\title{
Essentials of Glycobiology
}

\author{
(Varki, A., Cummings, R., Esko, J., Freeze, H., Stanley, P., Bertozzi, C., Hart, G., \\ and Etzler, M. (eds.) Cold Spring Harbor Laboratory Press, \\ Cold Spring Harbor, New York, 2009, 784 p., \$158)
}

DOI: $10.1134 / \mathrm{S} 0006297909090156$

The first edition of this book was published almost 10 years ago (see for book review Biochemistry (Moscow), No. 8, 2000). Glycobiology has advanced significantly during the past decade, and publishing of the second edition of this book is very timely. The book is dedicated to the memory of Rosalind Kornfeld and Roger Jeanloz, pioneers in the elucidation of glycan structure and function.

The book consists of 51 chapters, glossary, study guide, and subject index.

The first part, "General Principles", includes seven chapters that focus on the historical background of glycobiology and overview of major important subjects that form the of glycobiology: monosaccharides, major classes of glycoconjugates and glycans, their structure, biological role, diversity and site-specific glycosylation. The separate chapters of this part cover cellular organization of glycosylation, glycosyl transferases, and a genomic view of glycobiology.

The second part highlights structure and biosynthesis of glycans. There are data about structure of various types of $\mathrm{N}$-glycans, glycosphingolipids, proteoglycans and sulfated glycosaminoglycans, sialic acids, and hyaluronan.

The third part is devoted to organismal diversity. The evolution of glycan diversity in bacteria, fungi, viridiplantae, nematode, arthropoda, and deuterostomes is considered.

The fourth part includes chapters related to glycanbinding proteins. The first chapter covers history of discovery and classification of glycan-binding proteins. The following chapters discuss data about various types of lectins: R-, L-, P-, C-, and I-type lectins. Here also galectins, microbial lectins, and proteins that bind sulfated glycosaminoglycans are considered.

The fifth part is devoted to the role of glycans in physiology and disease. The role of glycans in glycoprotein quality control, their service as signaling molecules, and glycans in development and systematic physiology are discussed. Special chapters describe the role of glycans in bacterial, viral, and parasitic infections. Also there is information about genetic disorders of glycan degradation (lysosomal storage diseases), glycosylation, and disorders related to glycosylation changes in cancer.

The final, sixth part of book highlights methods and applications used for study of glycans. Methods where antibodies and lectins are applied for study of glycans, chemical and enzymatic synthesis of glycans and glycoconjugates, chemical tools for inhibiting glycosylation, and glycans in biotechnology and pharmaceutical industry are analyzed.

Each chapter contains a short list of references. The book also includes a list of fundamental monographs dealing with various aspects of glycobiology. At the end of book there is study guide consisting of very provocative questions related to each chapter covering the major ideas expressed in the text. A dictionary of terms and a subject index are very helpful for readers.

In general, the book is valuable not only for beginners and experts in glycobiology, but also for many biochemists, molecular biologists, biotechnologists, and physicians. 